\title{
Incidence and Characteristics of Facial Nerve Stimulation in Children With Cochlear Implants
}

\author{
Sharon L. Cushing, BScH, MD; Blake C. Papsin, MD, FRCS(C); Karen A. Gordon, MA, PhD
}

\begin{abstract}
Objectives: Electrical stimulation from a cochlear implant can spread beyond the auditory nerve. The aims of this study were to accurately measure facial nerve stimulation in pediatric implant users and to determine the characteristics and incidence of this unwanted activity. Part A consisted of a prospective study of a randomized sample of 44 pediatric implant users. Part B consisted of a retrospective analysis of 121 children with previously recorded electrically evoked auditory brainstem responses (EABR). Study Design and Methods: Responses were evoked by 3 electrodes along the implant array in three groups of children: 1) postmeningitic, 2) abnormal cochlea, and 3) neither. Intraoperative measures were obtained under anesthesia; all other recordings were completed in awake children. Results: Intraoperative recordings revealed large nonauditory responses in a number of channels, including the midline EABR. Under paralysis, these responses disappeared, and clear EABRs were recorded. Similarly, prospective postoperative electromyographic (EMG) responses from the facial nerve were found in more than $59 \%$ (26 of 44) of experienced implant users (Nucleus 24): $31 \%$ of postmeningitic children (4 of 13 ), $80 \%$ of those with abnormal cochlea (8 of 10 ), and $66 \%$ of those with neither (14 of 21). Retrospective analysis of previously recorded postoperative EABRs demonstrated facial nerve stimulation in $35 \%$ (42 of 121). In most cases, facial nerve stimulation occurred when levels were perceptually loud but comfortable. Conclusions: 1) Facial nerve potentials can be recorded using EMG in a large proportion of cochlear implant users at high levels of stimulation. 2) The EABR can be obscured in the presence of facial nerve stimulation and care should be taken to distinguish it from the EMG response, particularly when auditory brainstem activity is in question. 3)
\end{abstract}

From the Cochlear Implant Program, Department of Otolaryngology, The Hospital for Sick Children and the University of Toronto, Toronto, Canada.

Editor's Note: This Manuscript was accepted for publication May 16, 2006. Foundation.

Supported by a grant from the Physicians' Services Incorporated

Send correspondence to Sharon L. Cushing, BScH, MD, Cochlear Implant Laboratory, Room 6D08, The Hospital for Sick Children, 555 University Avenue, Toronto, Ontario, Canada M5G 1X8. E-mail: s.cushing@utoronto.ca

DOI: 10.1097/01.mlg.0000231303.85828.20
Use of surface EMG provides an additional objective measure to ensure the safe and comfortable use of cochlear implants. Key Words: Cochlear implants, pediatrics, facial nerve, electric stimulation.

Laryngoscope, 116:1787-1791, 2006

\section{INTRODUCTION}

Cochlear implantation has become a commonly performed procedure in children with severe to profound sensorineural hearing loss. As our experience has increased, the rate of complications with cochlear implantation has decreased. One potential complication is aberrant facial nerve stimulation, which can occur in either an immediate or delayed fashion. A number of conditions have been identified that appear to predispose patients to facial nerve stimulation. These include otosclerosis, otosyphilis, temporal bone fracture, closed head injury (both occurring pre- and postimplantation), osteoporosis, meningitis, hydrops, and many congenital malformations of the inner ear. Still, some patients develop facial nerve stimulation in the absence of any of these conditions. In their review of 100 adult patients receiving cochlear implants, Stoddart et al. found that the most common reason for an electrode to require deactivation was facial nerve stimulation. ${ }^{1}$

Currently, facial nerve stimulation is assessed only by visual detection of facial movements or by self-reported facial twitching. In most cases, unwanted facial movements in implant users can be eliminated by altering the programming strategy of the device or by deactivating or decreasing the stimulus intensity of the offending electrode(s). In general, this either does not or only minimally compromises hearing outcomes. However, in a smaller percentage of cases, facial movements cannot be eliminated while maintaining acceptable hearing. Likewise, these movements can be so bothersome that they render the implant useless leading to nonuse or even explantation. ${ }^{2,3}$

The lack of an objective measure of facial nerve stimulation increases the risk of unidentified activity in children. Children may not be able to comment on the presence of facial twitching, and it is possible that not all facial stimulation will be identified using the current clinical practice of visually inspecting the face for movement. In the present study, we asked if facial nerve stimulation 


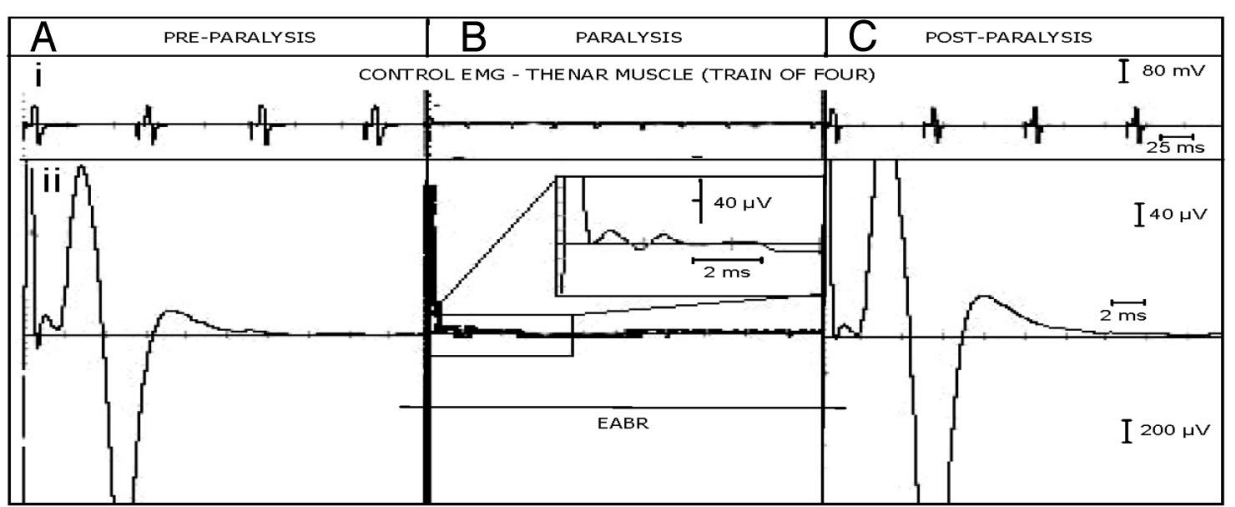

Fig. 1. Intraoperative recording demonstrating presence of a myogenic response from the facial nerve seen in the evoked auditory brainstem response (EABR) (ii) channels (A), which obliterates in the presence of a muscle relaxant allowing the underlying EABR to emerge (B). The myogenic, facial nerve response returns on reversal of the muscle relaxant (C). Control electromyography (train of four) of the thenar muscle was also performed (i). could be objectively measured through electrically evoked potentials and electromyography of the facial musculature. Our aims were to use the electrophysiological measures to: 1) identify the incidence of facial nerve activity in children; and 2) determine the relationship between facial nerve activity, perceptible facial twitching, and visually detectable movements of the face.

\section{MATERIALS AND METHODS}

This research was approved and overseen by the Hospital for Sick Children Research Ethics Board.

\section{Defining the Facial Nerve Response}

In an initial pilot study, facial nerve responses were first isolated from midline recordings in two patients at the time of cochlear implant surgery by comparing recordings made before, during, and after the administration of a single dose of paralytic agent $(0.5-0.6 \mathrm{mg} / \mathrm{kg}$ Rocuronium). The characteristic waveform of the myogenic response, described subsequently and shown in Figure 1, was always present in the midline recording channel. Facial nerve activity was confirmed by the concurrent presence of a response in at least one of the branches of the facial nerve monitored by additional recording channels. ${ }^{4}$ There was large individual variability in the pattern of specific facial nerve branch activation; therefore, the incidence of facial nerve stimulation was judged using the midline recording electrode for the purpose of the current article.

\section{Retrospective Group}

Midline recordings from 121 children were available to us as part of separate research projects conducted from January 1999 to June 2005. These recordings were either obtained intraoperatively with needle electrodes or postoperatively after device activation using a cortical surface electrode cap. These recordings were retrospectively reviewed for the presence or absence of the characteristic myogenic response from the facial nerve. These evoked auditory brainstem responses (EABR) had been recorded at loud but comfortable stimulus levels at or below the intensity required for a stapedial response. This was assessed using visual inspection of the stapes immediately after device insertion in the operating room by the implant surgeon for EABRs recorded at this time or through the use of impedance measurements for recordings completed postoperatively after device activation.

\section{Prospective Group}

All children implanted with a Nucleus 24 cochlear device (Cochlear Corporation, Melbourne, Australia) before March 2004 at the Hospital for Sick Children in Toronto, Canada, and with more than 12 months of implant experience were considered eligible to participate in the prospective arm of this study. A list of children meeting these selection criteria was compiled. Each child was assigned a number using a random number generator. The list of children was rank-ordered. Families were contacted using this randomly ordered list by the resident researcher (S.L.C.) who was blinded to the group and any known or previous reports of facial nerve stimulation. In total, 44 children were recruited to participate. These children were subdivided into three groups: children who, in their implanted ear, had 1) a normal cochlea (21 patients), 2) cochlear abnormalities (10 patients), and 3) acquired hearing loss after meningitis (13 patients). Of the 10 children in the prospective group who had abnormalities of the cochlea, 2 had common cavity deformities, 2 had dilated vestibular aqueducts, 3 had hypoplastic cochleas, and 3 had incomplete partitions, which includes the classic Mondini malformation. Informed consent was obtained from the parents and assent was obtained from the participant when possible.

Midline recordings were performed in combination with surface electromyographic (EMG) monitoring of four muscles innervated by the facial nerve (frontalis, orbicularis oculi, orbicularis oris, and platysma) in a random sample of pediatric implant users. Electrode configuration for a child using bilateral implants is shown in Figure 2. The midline recordings were made using a noninverting electrode $(+)$ at $\mathrm{C}_{\mathrm{Z}}(10-20$ system) and an inverting electrode $(-)$ on the ipsilateral earlobe. The time window of recording was -5 to 20 millisecond relative to stimulus onset (latency) and at least 200 sweeps were recorded and averaged using a NeuroScan recording platform (NeuroScan, El Paso, TX). Implant electrodes at the apical, mid, and basal portions of the
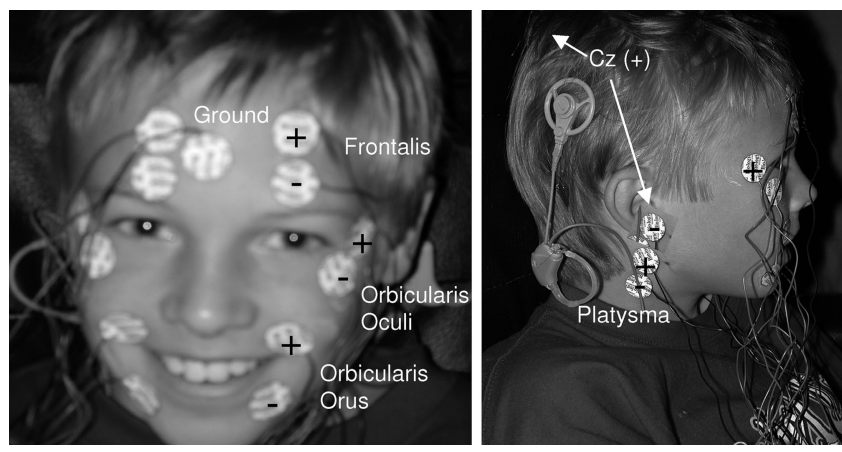

Fig. 2. Configuration of bilateral electrode placement for electromyography of facial musculature. $(+)$ positive electrodes, $(-)$ negative electrode, $\mathrm{C} z$ = evoked auditory brainstem response recording electrode. 
implanted array (typically 20,9, and 3, respectively) delivered single monopolar biphasic pulses of 25 to 100 microsecond/phase at a frequency of $11 \mathrm{~Hz}$. Electrical stimulation was provided at the uppermost end of the dynamic range of intensity as determined objectively using the electrically evoked stapedius reflex threshold (ESRT). The ESRT threshold has been shown to correlate with behaviorally determined levels of comfortable loudness. ${ }^{5}$ All children were instructed to report any sensations or discomforts. Stimulation levels were increased beyond the ESRT only if the children reported being comfortable with the stimulus intensity.

In total, recordings were made prospectively in 44 children who were $10.7 \pm 3.86$ years of age at the time of testing and who had $4.6 \pm 2.6$ years of implant experience.

\section{RESULTS}

\section{Defining the Facial Nerve Response}

In an initial pilot study, we clearly defined an electromyogenic response at the midline of the scalp evoked by electrical pulses in children using cochlear implants. This was accomplished prospectively at the time of surgical implantation in 2 children in whom the myogenic potentials were seen to be modulated by a single dose $(0.5$ to 0.6 $\mathrm{mg} / \mathrm{kg}$ ) of neuromuscular blocking agent (Rocuronium). Figure 1 shows that the characteristic waveform of the myogenic response recorded from the mid-line electrode in one child disappeared under paralysis leaving a clear recording of the underlying EABR waveform. This confirmed the myogenic nature of the initial waveform which reemerged upon reversal of paralysis. Control recordings of four stimuli on the thenar muscle are shown in the top panel of Figure 1; this known myogenic response similarly disappeared during paralysis and reappeared upon reversal.

\section{Incidence}

Retrospective analysis of midline recordings revealed the presence of myogenic responses from the facial nerve in $34 \%$ (42 of 121). Similarly, in the prospective group, myogenic responses from the facial nerve were detected in the midline recordings in more than 59\% (26 of 44) of experienced implant users (Nucleus 24): $31 \%$ (4 of 13) of postmeningitic children, $80 \%$ ( 8 of 10 ) of those with abnormal cochlea, and $66 \%$ (14 of 21) of children with normal cochleae. In most cases, facial nerve stimulation occurred when levels were perceptually loud but comfortable. Moreover, $18 \%$ ( 8 of 44 ) of the children in the prospective group had facial nerve stimulation thresholds that fell within the range of currents provided by the implant during daily use. Of these children, 4 had abnormal cochlea, 3 had normal cochlea, and one had acquired hearing loss subsequent to meningitis.

\section{Subclinical versus Clinical Stimulation}

Subclinical facial nerve stimulation was defined as the presence of a myogenic response from the facial nerve in the absence of either the sensation of movement reported by the implant user, or obvious twitching of the face reported by an observer. In Figure 3, the mean stimulation level at which a myogenic response was observed is compared to the mean levels at which sensation of movement was perceived and when observable movement of the face was visually observed. As shown in Figure 3, myogenic responses were recorded in 26 of 44 children (59\%) at stimulation levels of $224 \pm 13$ clinical units. Children did not report a sensation in their face until the stimulus intensity was increased $16.4 \pm 11$ clinical units (range, 1-50) above the threshold of the myogenic response. Of note, 17 children (39\% of the prospective group) reported sensations but 9 reported no perception of facial twitching. A further increase in stimulation levels was required to elicit visually observed movements of the face. Noticeable movements of the face occurred in 11 children (25\% of the prospective group) at $6.3 \pm 4$ clinical units (range, 3-15) above mean levels at which sensations were reported. In summary, Figure 3 demonstrates that the threshold for a myogenic response recorded in the midline is significantly lower than the threshold for the perception of facial twitching and the threshold for perceptible movement of the face.

In Figure 4, responses recorded in 3 children from the mid-line channel are shown with increasing stimulus intensity. Indicated are the threshold levels of the myogenic response and both the subjective (child report) and objective (visually observed) facial movements. The upper limit of stimulation levels (C-levels) programmed into the children's external cochlear implant equipment and their stapedius reflexes are also indicated. In children A and B, visually observed movements of the face did not occur within the levels programmed for their implants; however, sub-clinical myogenic responses were recorded and sensation was reported at levels within the children's programmed range. In child $\mathrm{C}$, subjective and objective movements of the face occurred at levels above the C-level. However, the subclinical myogenic response did occur at the child's C-level.
Fig. 3. Relationship between thresholds for myogenic facial nerve response using midline recordings and thresholds for a perceptible sensation of facial twitching and observable facial movement.

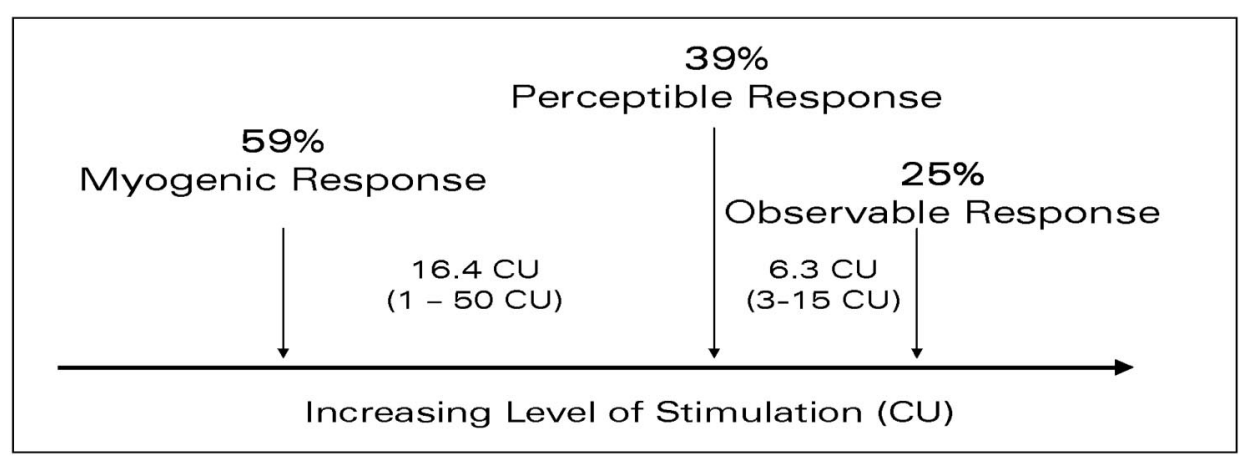



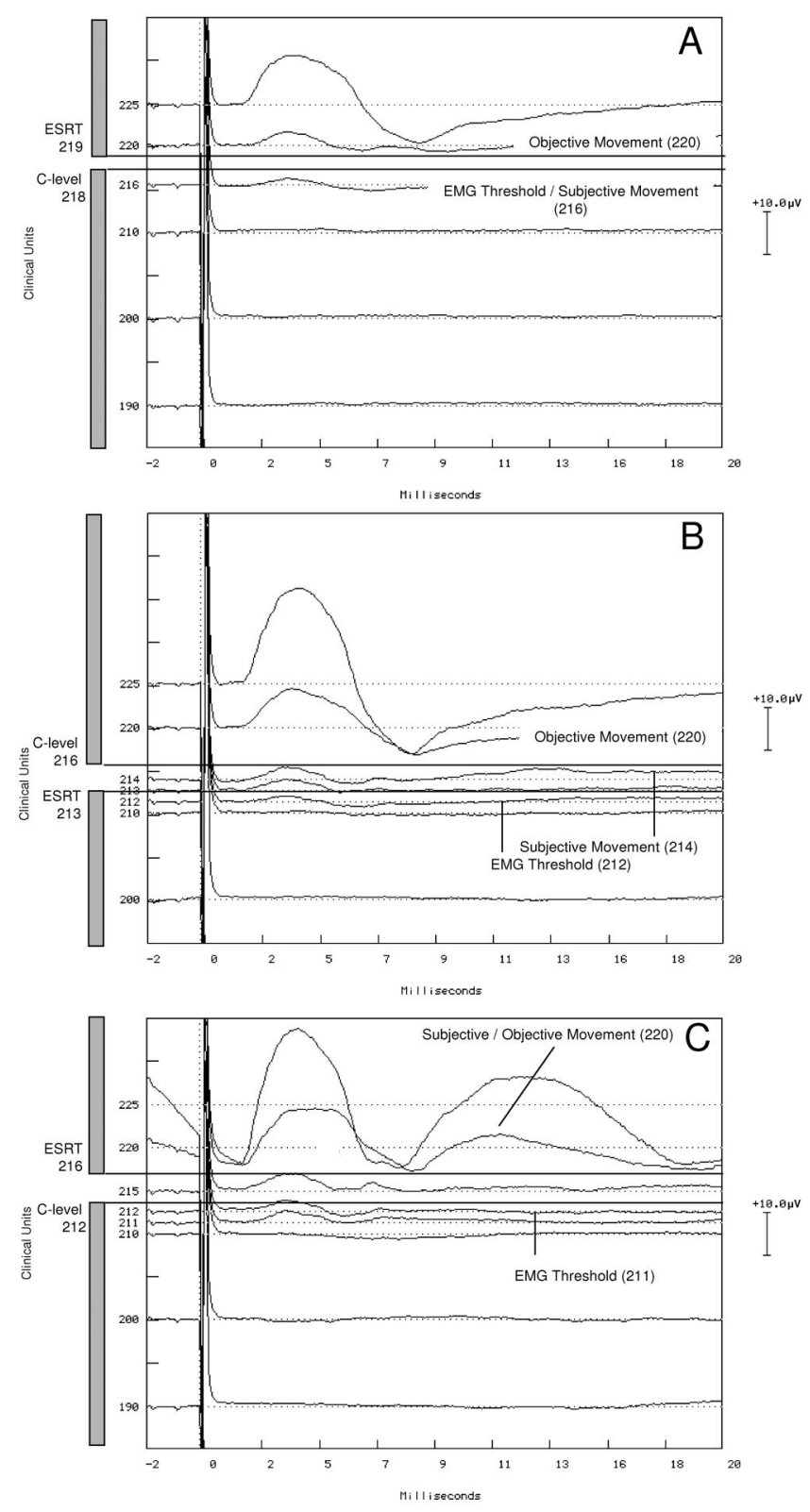

Fig. 4. Midline recordings (evoked auditory brainstem responses configuration) demonstrating myogenic response of the facial nerve for (A) implant electrode no. 3; (B) implant electrode no. 9; and (C) implant electrode no. 20. Electromyography thresholds and thresholds for subjective and objective movement are indicated. Evoked stapedial reflex threshold (ESRT) and comfort levels (C-levels) from current muscle action potential are indicated (solid lines).

\section{Electrode Location}

In both the prospective and retrospective recordings, three different electrodes were stimulated, one each at the apex, midportion, and base of the electrode array (electrodes 20,9, and 3). When myogenic responses from the facial nerve were present, they were evoked universally across the array and not limited to electrodes within a specific region.

\section{DISCUSSION}

Programming electrical stimulation levels in cochlear implant users involves a careful balancing between provi- sion of an optimal dynamic range of intensities and unintended percepts, including facial nerve activation. This is most challenging in the pediatric population who may not identify or have the language to express the sensation of facial twitching. Therefore, in this population, we often rely solely on visual detection of facial movement because no objective measure of electrically evoked facial nerve stimulation has been previously reported. The waveforms identified and characterized in the present study provide a much needed base on which to build our understanding of this serious complication of cochlear implantation.

The sequelae of facial nerve stimulation ranges from significant discomfort to suboptimal use of cochlear implants, and often entails significant time spent in reprogramming. At the extreme, facial nerve stimulation can force nonuse or even explantation of the device.

\section{Incidence}

Facial nerve stimulation in the setting of cochlear implantation was initially reported by Cohen and Hoffman in 1988. A survey review of surgeons performing cochlear implants elicited a total of four of 459 cases of facial nerve stimulation $(0.9 \%) .6$ In a further review of the Cochlear Corporation database, this same group reported that 101 of 4,969 implant patients experienced facial nerve stimulation $(2.03 \%){ }^{7}$ In the pediatric setting, Kempf et al. reported that 11 of 366 implanted children $(3.0 \%)$ developed facial nerve stimulation as a delayed complication. ${ }^{8}$ An additional analysis of the Cochlear Corporation database by Kelsall et al., through personal communication in 1997, revealed a rate of facial nerve stimulation in approximately $3 \%$ of adults (102 of 3,502$)$ and $1 \%$ of children $(26$ of 2,442$) .{ }^{9}$ The incidence of facial nerve stimulation in the setting of cochlear implantation across all case series in the literature was tabulated in a 1998 review and the rates varied from $0.9 \%$ to $14.6 \% .10$ The current study suggests that facial nerve stimulation in pediatric implant users is significantly higher, ranging from $31 \%$ to $78 \%$. The difference may be that the incidence numbers in the literature are based on either verbal reports of patients describing twitching of the facial musculature or facial twitching obvious to the observer, usually the audiologist as opposed to EMG recording as used in the current study.

\section{Clinical versus Subclinical Facial Nerve Stimulation}

As shown in the current study, there is a large difference in the incidence of facial nerve stimulation when electrophysiological recordings are compared with patient reports or observation of facial movement. As shown in Figures 3 and 4, the midline recordings of myogenic responses can detect facial nerve activity at lower stimulation levels than those required to elicit perceptible or observable facial twitching.

\section{Facial Nerve Stimulation and Cochlear Malformations}

The majority (78\% [7 of 9]) of patients with cochlear malformations were noted to have inducible facial nerve stimulation in our series. The presence of cochlear abnormalities has been reported to be a risk factor for aberrant 
facial nerve stimulation, interfering with programming of optimal stimulation levels, compared with children with normal cochlea. ${ }^{11}$ Hoffman et al. examined the surgical and rehabilitative issues raised by implantation in children with cochlear dysplasia. In this group of patients, facial nerve anatomy was found to be anomalous in $16 \% .^{12}$ Postoperative facial nerve stimulation can certainly occur because of an atypical course of the facial nerve. In their study of temporal bone histopathology related to cochlear implantation in the congenitally malformed bony cochlea, they found that in $43 \%$ of cases, there was an abnormal course of the facial nerve. ${ }^{12}$ Dehiscence or abnormal course of the facial nerve running near the oval window or on the promontory both affect the insertion of the electrodes, the risk of facial nerve injury, as well as the risk of postoperative aberrant facial nerve stimulation. In children with congenital cochlear dysplasia, electrically evoked facial nerve stimulation has been reported in nine cases. ${ }^{13}$ In addition, it is felt that the increased frequency of shortened cochlear ducts in this population may lead to incomplete electrode insertion, further increasing the risk of facial nerve stimulation. ${ }^{3}$ Therefore, the risk of facial nerve stimulation in children with congenital inner ear deformities is higher for a number of reasons and this is reflected in our study population. None of the children in the prospective study, however, had any identified anomalies of the facial nerve on either computed tomography or magnetic resonance imaging.

\section{Facial Nerve Stimulation in Meningitis}

In the present study, children who were implanted following meningitis displayed the lowest incidence of facial nerve stimulation (31\% [4 of 13]) of the 3 groups. A number of reports in the literature identify meningitis as a risk factor for facial nerve stimulation. In their 1997 review of the Cochlear Corporation database, Kelsall et al. noted that all pediatric patients ${ }^{3}$ found to have facial nerve stimulation acquired hearing loss after meningitis. ${ }^{9}$ In all three children, cochlear ossification was encountered at the time of surgery and, as a result, all underwent a surgical drillout procedure. ${ }^{9}$ In a similar series, Kempf et al. note that the majority of the children with facial nerve stimulation had an underlying etiology of meningitis for their hearing loss. ${ }^{8}$ This trend, however, was not reflected in our current study. This result may be a consequence of our institution's more recent practice of implanting children as early as possible before the occurrence of cochlear ossification with none of the children studied requiring a cochlear drillout.

\section{CONCLUSIONS}

Results of the current study indicate that the incidence of facial nerve stimulation in pediatric cochlear implant users is much higher than what is previously reported in the literature. Self-reporting and/or visual detection of facial nerve stimulation appears to be inadequate, especially in the pediatric setting. Although it is unclear what the sequelae of chronic subclinical stimulation of the facial nerve are, this is likely a side effect of cochlear implant stimulation that is best avoided. In future research, we hope to examine how the thresholds for facial nerve stimulation may change with time and aging by following children with known facial nerve stimulation longitudinally.

\section{Acknowledgments}

Special thanks to Patricia Fuller and the audiologists in the Cochlear Implant Program at the Hospital for Sick Children. Thanks also to Samuel Strantzas and Dr. Homan Arkia for their help with the intraoperative recordings.

\section{BIBLIOGRAPHY}

1. Stoddart RL, Cooper HR. Electrode complications in 100 adults with multichannel cochlear implants. J Laryngol Otol 1999;113:18-20.

2. Kumar A, Mugge R, Lipner M. Surgical complication of cochlear implantation: a report of three cases and their clinical features. Ear Nose Throat J 1999;78:913-919.

3. Maas SF, O'Driscoll M, Mawman D, et al. Explantation of a nucleus multichannel cochlear implant a re-implantation into the contralateral ear. A case report of a new strategy. $J$ Laryngol Otol 1996;110:881-883.

4. Cushing SL, Gordon KA, Papsin BC. Clinical and Subclinical Facial Nerve Stimulation in Children With Cochlear Implants. Conference on Implantable Auditory Prostheses; 2005:199.

5. Gordon KA, Papsin BC, Harrison RV. Toward a battery of behavioral and objective measures to achieve optimal cochlear implant stimulation levels in children. Ear Hear 2004;25:447-463.

6. Cohen NL, Hoffman RA, Stroschein M. Medical or surgical complication related to the Nucleus multichannel cochlear implant. Ann Otol Rhinol Laryngol Suppl 1988;135:8-13.

7. Hoffman RA, Cohen NL. Complication of cochlear implant surgery. Ann Otol Rhinol Laryngol 1995;104:420-422.

8. Kempf HG, Johann K, Lenarz T. Complication in pediatric cochlear implant surgery. Eur Arch Otorhinolaryngol 1999;256:128-132

9. Kelsall DC, Shallop JK, Brammeier TG, et al. Facial nerve stimulation after Nucleus 22-channel cochlear implantation. Am J Otol 1997;18:336-341.

10. Bigelow DC, Kay DJ, Rafter KO, et al. Facial nerve stimulation from cochlear implants. Am J Otol 1998;19:163-169.

11. Papsin BC. Cochlear implantation in children with anomalous cochleovestibular anatomy. Laryngoscope 2005;115: $1-26$.

12. Hoffman RA, Downey LL, Waltzman SB, et al. Cochlear implantation in children with cochlear malformations. Am J Otol 1997;18:184-187.

13. Graham JM, Phelps PD, Micheals L. Congenital malformation of the ear and cochlear implantation in children: review and temporal bone report of common cavity. J Laryngol Otol 2000;114:1-14. 